\title{
An unusual case of invasive aspergillosis in an immunocompetent individual
}

\author{
Afsal P Mohammed, ${ }^{1}$ Pushwinder Dhunputh, ${ }^{1}$ Raghuvaran Chiluka, ${ }^{2}$ \\ Shashikiran Umakanth ${ }^{1,2}$
}

\section{Correspondence to}

Professor Shashikiran

Umakanth,

shashikiranu@gmail.com

Accepted 31 May 2015

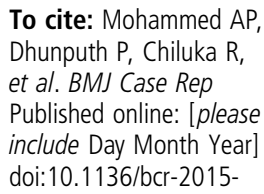

\section{${ }^{1}$ Department of Medicine,
Melaka-Manipal Medical \\ Department of Medicine,
Melaka-Manipal Medical College, Manipal, Karnataka, India \\ ${ }^{2}$ Department of Medicine, Dr TMA Pai Hospital, Udupi, Karnataka, India}

\section{SUMMARY}

Invasive aspergillosis is a highly lethal opportunistic infection that poses a significant threat to immunocompromised patients. With studies suggesting that the incidence of this disease is increasing, and mortality rates remain high, early diagnosis and treatment are very important to improve patient survival. We present the case of a 33-year-old immunocompetent woman who presented with a history of cough and severe breathlessness, and was diagnosed to have invasive aspergillosis. This case emphasises the importance of maintaining a high index of suspicion and also of remembering that invasive aspergillosis is no longer only a disease of immunocompromised individuals. In addition, this case tells us that aspergillosis is one of the new emerging infections in intensive care units.

\section{BACKGROUND}

Aspergillus spp are ubiquitous fungi in the external environment and inhalation of infectious conidia is a common event; however, tissue invasion, or invasive aspergillosis, is uncommon. Invasive aspergillosis occurs most frequently in the setting of immunosuppression and is a rapidly progressive, frequently fatal disease. Patients at risk include those with prolonged neutropaenia, recipients of haematopoietic stem cell transplants or solid-organ transplants and those with advanced AIDS or chronic granulomatous disease. Also at risk, but rarely seen, are patients with serious autoimmune disorders being treated with intensive immunosuppressive regimens.

Despite the usual presentation, aspergillosis may also, in rare situations, occur in immunocompetent individuals, which compelled us to highlight this case.

\section{CASE PRESENTATION}

A 33-year-old housewife with no premorbidities presented with sudden onset of breathlessness after a 4-day history of cough and fever. On examination, she was febrile and tachypnoeic, with oxygen saturation of $84 \%$ on high-flow oxygen. Respiratory system examination showed bronchial breath sounds in left supraclavicular and interscapular areas; all other systems were within normal limits. Hence a clinical diagnosis of left upper lobe pneumonia with acute respiratory distress syndrome (ARDS) was initially made, based on the presentation.

\section{INVESTIGATIONS}

Laboratory investigations showed normal white cell counts (WCC) with mildly elevated erythrocyte sedimentation rate $(31 \mathrm{~mm} / \mathrm{h})$ and mild elevation of aspartate aminotransferase/alanine transaminase (144/152 U/L) levels. Other routine blood parameters were normal. Initial workup for fever, including malaria, dengue, scrub typhus and leptospirosis tests, were negative, thereby ruling out the other common causes of ARDS prevalent in the locality. Hepatitis B surface antigen and HIV were also negative. Chest X-ray showed left hemithorax haziness (figure 1). ECG showed sinus tachycardia and two-dimensional echocardiography was normal. Ultrasound study of the abdomen was normal. Arterial blood gases showed respiratory alkalosis with $\mathrm{PO}_{2} / \mathrm{FiO}_{2}$ of 121 . A clinical diagnosis of left upper lobe pneumonia with ARDS was considered, and the patient was started on broad-spectrum antibiotics. She was also started on oseltamivir, after collecting respiratory samples, in view of the possibility of H1N1 influenza, which is commonly seen at that time of the year in our community.

As the patient continued to be tachypnoeic and subsequent investigations showed increasing WCC, she was electively intubated and antibiotics were escalated. Meanwhile, blood and urine cultures were reported as sterile, and viral studies were negative for influenza and other common respiratory viruses. Sputum samples for acid-fast bacilli were reported as negative. On the fourth day of

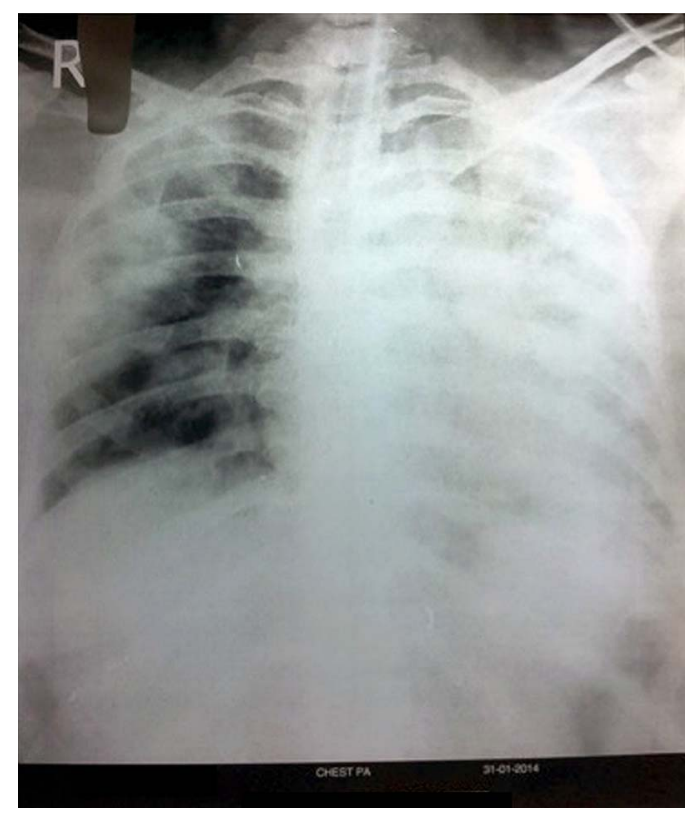

Figure 1 Left hemithorax homogeneous opacity. 


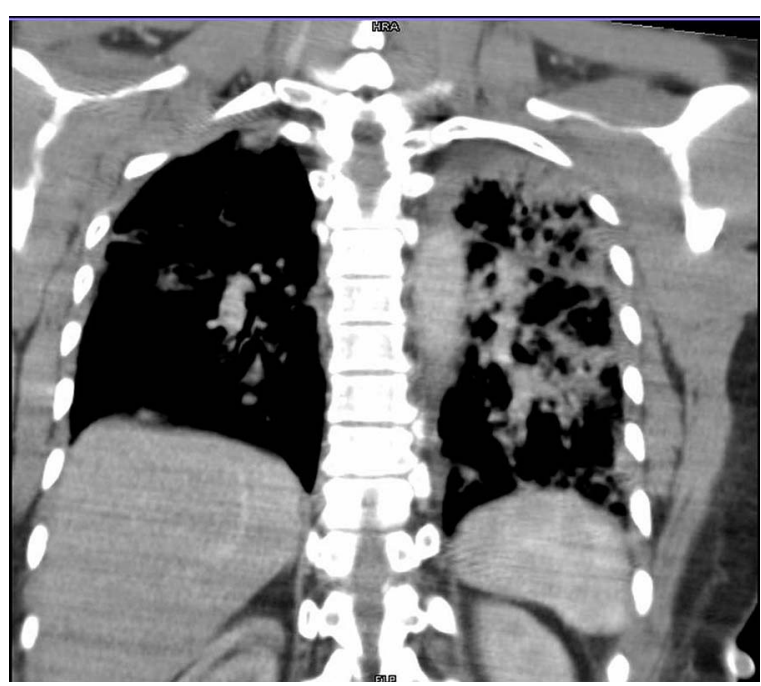

Figure 2 Focal and patchy areas of consolidation (with areas of breakdown), ground glass attenuation and a few random nodules diffusely scattered in both lungs, the largest area being in left upper lobe. Bilateral mild pleural effusion.

admission, the patient's sputum culture was reported as positive for Aspergillus fumigatus. Since we considered that as possibly a normal upper respiratory commensal, a repeat culture of endotracheal aspirate was sent. When that was also reported as positive for the same organism the next day, the patient was started on intravenous voriconazole with intravenous caspofungin, after sending for a repeat blood culture and Aspergillus galactomannan antigen assay.

CT scan of the thorax was suggestive of an acute infective aetiology in both lungs, predominantly in the left upper lobe (figure 2). A CT scan of the osteomeatal complex was carried out to look for the source of infection from paranasal sinuses, and was normal (figure 3). PCR for Pneumocystis jirovecii, antinuclear antibody profile, immunoglobulin and complement levels, were within normal limits, ruling out any immunocompromised status. A transbronchial lung biopsy could not be performed as we do not have that facility in our setting.

After starting voriconazole and caspofungin, the patient started showing improvement clinically; investigation reports also showed signs of recovery. Aspergillus galactomannan antigen ELISA was reported as positive, confirming evidence of

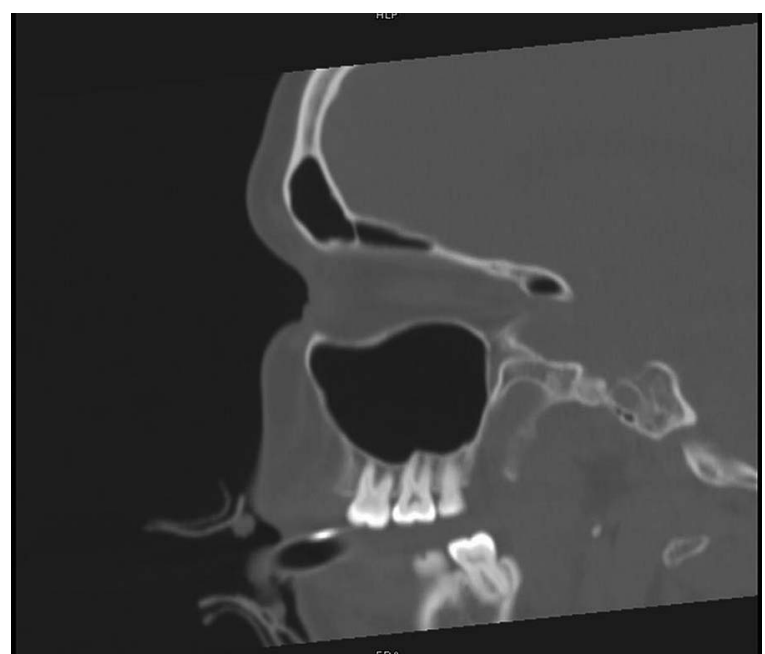

Figure 3 Normal CT of the osteomeatal complex. invasive aspergillosis. The patient was extubated after 7 days of intravenous antifungal therapy and repeat sputum culture was sterile. She was discharged from the hospital uneventfully and followed up after 2 weeks, with blood counts and chest X-ray, which were completely normal.

\section{DIFFERENTIAL DIAGNOSIS}

- Community acquired pneumonia

- ARDS

\section{TREATMENT}

The standard regimen for initial therapy of invasive aspergillosis diagnosed by culture, galactomannan antigen or histopathology, is a combination therapy with azole and an echinocandin. In our patient, we choose to give the above regimen using voriconazole and caspofungin; the patient responded well to the treatment, with no complications.

\section{OUTCOME AND FOLLOW-UP}

The patient recovered completely after the appropriate regimen for aspergillosis and on follow-up after 2 weeks of discharge she was asymptomatic and her chest X-ray was completely normal.

\section{DISCUSSION}

As illustrated in our case, invasive aspergillosis is challenging to diagnose. The presentation is based on sites of infection, with lungs being the most common organ. Other less common sites include the sinuses, gastrointestinal (GI) tract or skin. Invasive pulmonary aspergillosis presents with non-specific symptoms usually mimicking bronchopneumonia. ${ }^{1}$ It is also important to note that the classical 'halo' sign seen on CT scan of the thorax is common in immunocompromised hosts, but often not noticed in immunocompetent hosts. ${ }^{2}$ This makes the diagnosis even more challenging in immunocompetent hosts.

Invasive pulmonary aspergillosis most often disseminates to the brain, however, it may also spread to the skin, thyroid, bone, kidneys, liver, GI tract, eyes or heart. Histopathological examination with microbiological confirmation remains the gold standard in diagnosis; however, it is often difficult or not feasible to obtain a biopsy specimen, as in our case. The histological findings characteristic of aspergillosis are septated hyaline hyphae with acute angle $\left(45^{\circ}\right)$ dichotomous branching visible with H\&E or Grocott's methenamine silver staining. ${ }^{3}$

Blood cultures are rarely positive and sputum cultures are of limited value. Aspergillus spp may be found in the sputum in up to $15 \%$ of healthy people, however, up to $70 \%$ of those confirmed with invasive aspergillosis have a negative sputum result. The isolation of Aspergillus spp from bronchoalveolar lavage fluid from immunosuppressed patients is highly indicative of invasive aspergillosis ( $97 \%$ specificity), but is only positive in $50-58 \%$ of patients. ${ }^{4}$ In addition to the lack of sensitivity, the diagnosis may be delayed, since it may take a few days to grow from culture. Therefore, it is usual to receive negative results, especially in the setting of an immunocompetent host, but a high index of suspicion made us workup this case for detection of aspergillosis.

Owing to the limitations of the conventional diagnostic techniques, other modalities for diagnosis have been developed and are an area of current research. The two commercially available assays are galactomannan and $\beta-(1,3)$-d-glucan (BDG), both of which are components of the fungal cell wall. The galactomannan assay is relatively specific for invasive aspergillosis. In a meta-analysis, the galactomannan assay had a sensitivity of $71 \%$ and a specificity of $89 \%$. The BDG assay also detects other 
invasive fungal diseases, with the most complete study showing a sensitivity of $64 \%$ and specificity of $84 \% .^{5}$ Currently, these tests can be used in combination with several diagnostic techniques to rule out a fungal infection in patients at risk. Techniques

\section{Patient's perspective}

- Lung infection with invasive aspergillosis is a rare condition in those without diabetes, cancer and other such serious conditions.

- I was lucky to be diagnosed with this disease at the right time so that correct treatment could be started for my recovery. Those initial few days in the ICU immediately before and during mechanical ventilation were very scary.

\section{Learning points}

- Invasive aspergillosis has always been considered as an infection expressly of immunocompromised individuals.

- It is an emerging infection in the intensive care units and must be in our spectrum of differential diagnosis in immunocompetent individuals as well, especially when the patient does not respond to initial treatment.

- The classical 'halo' sign of invasive aspergillosis on CT scan of the thorax is often absent in immunocompetent hosts and diagnosis requires a very high degree of suspicion.

- Voriconazole in combination with an echinocandin shows good response in invasive aspergillosis. using fungal nucleic acid detection, mostly based on PCR, still remain investigational.

Early initiation of antifungal therapy in patients with highly suspected invasive aspergillosis is warranted while a diagnostic evaluation is conducted. Based on the largest randomised controlled trial, it is recommended that voriconazole ${ }^{6}$ be used for the primary treatment of invasive aspergillosis. It has also been used in many cases of extrapulmonary and disseminated infection, inferring it is effective in these cases as well. Alternatives to voriconazole are the formulations of amphotericin B, itraconazole and caspofungin.

Acknowledgements Dr Chiranjay Mukhopadhyay, Professor and Head, Department of Microbiology, Kasturba Medical College, Manipal, Karnataka, India. Dr Seema Shetty, Associate Professor, Department of Medicine, Melaka-Manipal Medical College, Madhav Nagar, Manipal, Karnataka, India.

Competing interests None declared.

Patient consent Obtained.

Provenance and peer review Not commissioned; externally peer reviewed.

\section{REFERENCES}

1 Clancy CJ, Nguyen MH. Acute community-acquired pneumonia due to Aspergillus in presumably immunocompetent hosts: clues for recognition of a rare but fatal disease. Chest 1998:114:629-34.

2 Zhang R, Wang S, Lu H, et al. Misdiagnosis of invasive pulmonary aspergillosis: a clinical analysis of 26 immunocompetent patients. Int I Clin Exp Med 2014;7:5075-82.

3 Kousha M, Tadi R, Soubani AO. Pulmonary aspergillosis: a clinical review. Eur Respir Rev 2011;20:156-74

4 Nguyen $\mathrm{MH}$, Jaber $\mathrm{R}$, Leather $\mathrm{HL}$, et al. Use of bronchoalveolar lavage to detect galactomannan for diagnosis of pulmonary aspergillosis among nonimmunocompromised hosts. J Clin Microbiol 2007;45:2787-92.

5 Maertens J, Maertens V, Theunissen K, et al. Bronchoalveolar lavage fluid galactomannan for the diagnosis of invasive pulmonary aspergillosis in patients with hematologic diseases. Clin Infect Dis 2009:49:1688-93.

6 Steinbach WJ, Juvvadi PR, Fortwendel JR, et al. Newer combination antifungal therapies for invasive aspergillosis. Med Mycol 2011:49(Suppl 1):S77-81.

Copyright 2015 BMJ Publishing Group. All rights reserved. For permission to reuse any of this content visit http://group.bmi.com/group/rights-licensing/permissions.

BMJ Case Report Fellows may re-use this article for personal use and teaching without any further permission.

Become a Fellow of BMJ Case Reports today and you can:

- Submit as many cases as you like

- Enjoy fast sympathetic peer review and rapid publication of accepted articles

- Access all the published articles

- Re-use any of the published material for personal use and teaching without further permission

For information on Institutional Fellowships contact consortiasales@bmjgroup.com

Visit casereports.bmj.com for more articles like this and to become a Fellow 